\title{
Parameter Estimation in Convection-Diffusion Model
}

\author{
XIAOYANG ZHENG ${ }^{1}$, SHU CHEN ${ }^{2}$, JIANGPING HE ${ }^{2}$,

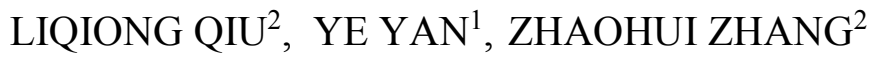 \\ ${ }^{1}$ College of Artificial Intelligence, Chongqing University of Technology, Chongqing, CHINA \\ ${ }^{2}$ College of Science, Chongqing University of Technology, Chongqing, CHINA
}

\begin{abstract}
This paper describes a special one-dimensional convection-diffusion equation and analyzes two types of difference schemes. Numerical solutions of the two difference methods for this equation are implemented to estimate the parameters of the velocity component of the fluid and the diffusion coefficient. Good results of parameters estimated are not achieved because of the larger approximation errors by the difference schemes. Then multiple linear regression is applied to estimating the corresponding parameters by using the analytical solution of this special equation. By this means, the better estimated values of the parameters are obtained.
\end{abstract}

Key-Words: - Convection-diffusion equation; finite difference method; multiple linear regression; parameter estimation.

Received: August 29, 2020. Revised: December 4, 2020. Accepted: December 18, 2020. Published: December 29, 2020.

\section{Introduction}

The convection-diffusion equation arises in many important applications, such as conservative solute transport in porous media, mass transfer and heat transfer etc. [1-8]. This is one of the most frequently used models in science and engineering. For example, the model describes how the concentration of one or more substances (e.g., pollutants) distributed in a medium changes under the influence of three processes, namely, convection, diffusion, and reaction for groundwater pollution and air pollution in environmental science [5-11].

The goal of this article is to estimate the parameters of the convection and diffusion terms in the one-dimensional convection-diffusion equation as the form [3]

$\frac{\partial u}{\partial t}=-v \frac{\partial u}{\partial x}+\mu \frac{\partial^{2} u}{\partial x^{2}} \quad x \in R, \quad 0<t \leq T$

where $u(x, t)$ is unknown and denotes the concentration of the pollutant in the medium, $v$ and $\mu$ are parameters of velocity component of the fluid and diffusion coefficient, respectively. Its corresponding initial condition is a Gaussian distribution of the substance in an infinite medium.

It is known that it is very difficult to obtain analytical solution to Eq.(1) when initial and boundary conditions are complicated, even with constant parameter coefficients [4-5]. Generally speaking, we can develop numerical solution of Eq.(1) to estimate the parameters, which means to optimally adapt models to given data by determining their parameter values such that the deviation of model and data is minimized in a suitable norm.

Fortunately, a lot of numerical techniques for Eq.(1) are by now well developed such as finite differences, finite elements, spectral procedures, wavelet-Galerkin methods, discontinuous Galerkin methods and Graphical methods etc. [5-16]. In this paper, we concentrate on the parameters estimation by using the finite difference approach and the multivariate linear regression method, respectively.

This paper is organized as follows: In Section 2, two types of finite differences are introduced and applied to solving numerically Eq.(1). Section 3 utilizes the least squares approach to estimate the parameters in Eq.(1) by using the numerical solutions. In Section 4, the multivariate linear regression method is implemented to estimate the parameters $v$ and $\mu$ in Eq.(1) with analytical solution situation. Conclusions of the parameter estimation methods for Eq.(1) and some suggestions for future research are given at the end in Section 5. 


\section{Two types of difference schemes for convection-diffusion equation}

In this section, the two finite differences such as forward-time centered-space and upwind difference schemes are described and compared for solving Eq.(1) with constant coefficients.

.For clarity, we begins with a discretization of the domains $[0,1] \subset R$ and $[0, T]$ of the independent the space variable $x$ and the time variable, respectively. We subdivide the interval $[0,1]$ in ${ }^{t}$ to $M$ sub-intervals and the interval $[0, T]$ into $N$ subintervals such that $M h=1$ and $N \tau=T$, where $h$ is space step and $\tau$ denotes time step, respectively. Then, the grid points $\left(x_{j}, t_{n}\right)$ are defined as $x_{j}=j h, \quad j=0,1,2, \cdots, M, t_{n}=n \tau, \quad n=0,1,2, \cdots, N$.

Note that the numerical solution is to find the difference approximation values $u_{j}^{n}$ of $u(x, t)$ at the points $\left(x_{j}, t_{n}\right)$.

\subsection{Forward-time centered-space difference method}

The simplest difference scheme of all is the forward-time centered-space discretization of the full Eq.(1), i.e.

$\frac{u_{j}^{n+1}-u_{j}^{n}}{\tau}=-v \frac{u_{j+1}^{n}-u_{j-1}^{n}}{2 h}+\mu \frac{u_{j+1}^{n}-2 u_{j}^{n}+u_{j-1}^{n}}{h^{2}}$

which can be transformed into an explicit finite difference scheme of the form

$u_{j}^{n+1}=(\beta+\alpha / 2) u_{j-1}^{n}+(1-2 \beta) u_{j}^{n}+(\beta-\alpha / 2) u_{j+1}^{n}$

where $\alpha=v \tau / h$ and $\beta=\mu \tau / h^{2}$. Obviously, the truncation error of the (3) is $\mathrm{O}\left(\tau+h^{2}\right)$ and the corresponding stability requirement satisfies $\alpha^{2} / 2 \leq \beta \leq 0.5$, i.e. the choice of extremely small values for $\tau$ (Isenberg, 1972). Furthermore, its implicit form is demonstrated that

$u_{j}^{n+1}=\frac{1}{1+2 \beta}\left[(\alpha / 2+\beta) u_{j-1}^{n+1}+u_{j}^{n}+(-\alpha / 2+\beta) u_{j+1}^{n+1}\right]$

which is obtained by the backward-difference for the time derivative and centered-difference forms for all spatial derivatives.

In addition, the explicit form means to solving the value of grid-node in $n+1$ time level by using the values of grid-nodes in $n$ time level. Although this implicit difference technique needs to solve a system of algebraic equations for each time step, it is unconditionally von Neumann stable [3-7]. Consequently, there is no restriction to selecting the size of time step $\tau$.

\subsection{Upwind difference scheme}

The discretization of the upwind difference scheme can be specifically described as

$\frac{u_{j}^{n+1}-u_{j-1}^{n}}{\tau}=v \frac{u_{j}^{n}-u_{j-1}^{n}}{h}+\mu \frac{u_{j+1}^{n}-2 u_{j}^{n}+u_{j-1}^{n}}{h^{2}}, \quad v>0$

which is specifically interpreted as the forwarddifference for the time derivative, backwarddifference for the convection term and centereddifference for the diffusive derivatives, respectively. Similarly, the explicit upwind difference has the below form

$$
u_{j}^{n+1}=(1-\alpha-2 \beta) u_{j}^{n}+(\alpha+\beta) u_{j-1}^{n}+\beta u_{j+1}^{n}, \quad v>0
$$

The corresponding truncation error is $\mathrm{O}(\tau+h)$ and the stability condition is $\alpha(\alpha-1) / 2 \leq \beta \leq(1-\alpha) / 2$ for the scheme. When the backward-difference, backward difference and centered-difference are applied to the time, convection diffusive derivatives in Eq.(1) respectively, the implicit difference form is obtained as

$u_{j}^{n+1}=\frac{1}{1+\alpha+2 \beta}\left[(\alpha+\beta) u_{j-1}^{n+1}+u_{j}^{n}+\beta u_{j+1}^{n+1}\right]$

\section{Parameter estimation by the difference schemes}

Generally, mathematical models are often developed to describe natural phenomena, but the models contain a number of parameters that cannot be measured or calculated. Consequently, parameter estimation is an important scientific problem in various fields [1-7].

In this Section, we concentrate on estimation of the parameters $v, \mu$ in Eq.(1) by using the difference solutions and the least squares approach. For simplicity, we first consider the convectiondiffusion with exact solution as [3]

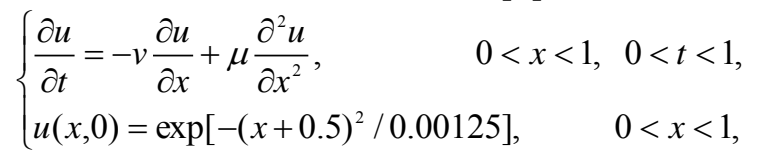

where the parameters $v=1, \mu=0.01$ and its exact solution is

$u(x, t)=\frac{0.025}{\sqrt{0.000625+0.02 t}} \exp \left[-(x+0.5-t)^{2} /(0.00125+0.04 t)\right]$

and the boundary conditions are decided by the analytical solution (10).

In this example, the steps of the difference grid points are set as $\Delta x=h=0.02, \Delta t=\tau=0.004$ for the spacial and time steps, respectively. Then Eq.(9) is numerically solved by the forward-time centeredspace method and the upwind difference scheme, respectively. In Figure 1, the absolute errors between the forward-time centered-space difference 
and the exact solutions are described at the grid points $51 \times 251$. Additionally, the $L_{1}$ error of this scheme is 0.0086 .

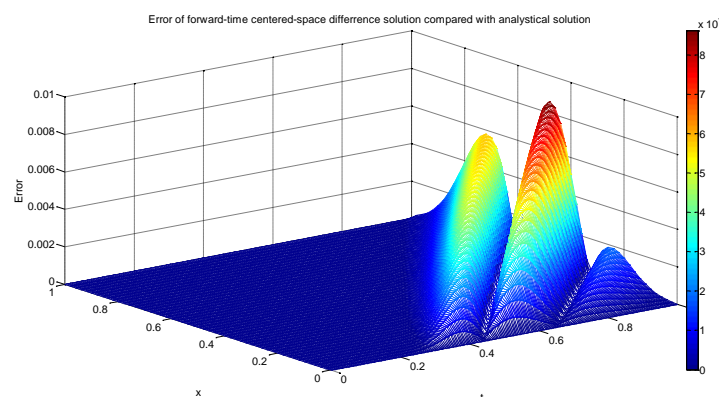

Figure 1. The error of forward-time centered-space solution compared with exact solution

Accordingly, Figure 2 depicts the comparisons of the numerical and the exact solutions at time $t=0.7$, $t=0.8$, respectively.

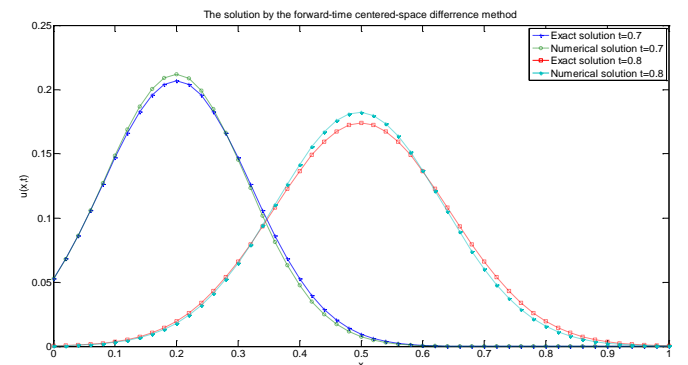

Figure 2. The solution by the forward-time centeredspace difference scheme with different time

Similarly, Figure 3 demonstrates the absolute errors between the upwind difference and the exact solutions and its $L_{1}$ error of this scheme is 0.0244 .

In Figure 4, the comparisons of the upwind difference solution and the exact solution at time $t=0.7, t=0.8$ are shown, respectively.

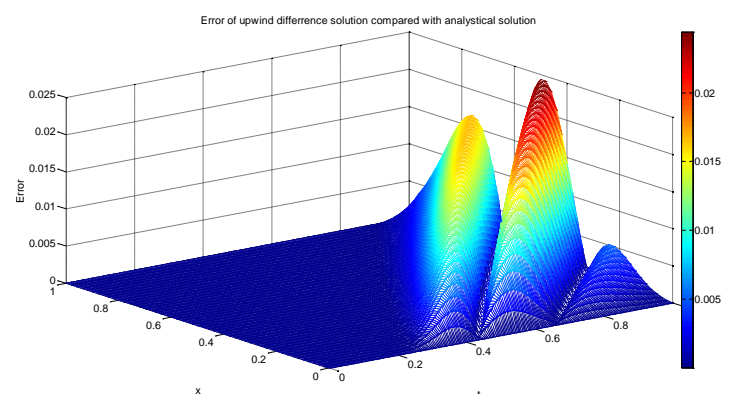

Figure 3. The error of the upwind difference solution compared with exact solution

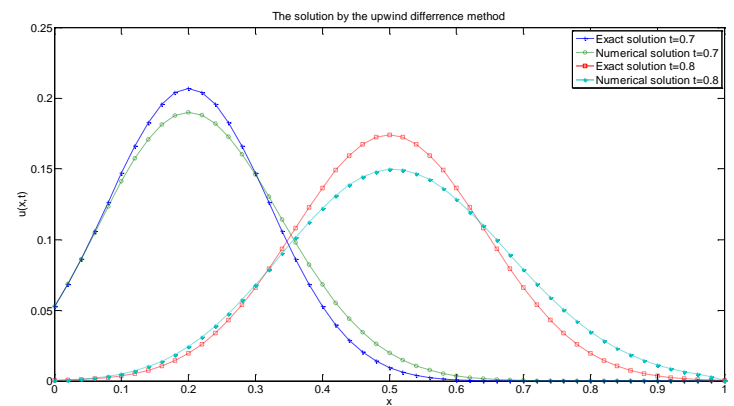

Figure 4. Comparison of the numerical and exact solutions with different time

Now we turn to the parameter estimation and we firstly use the values $u\left(x_{j}, t_{n}\right)$ of the analytical solution at the grid points $\left(x_{j}, t_{n}\right)$ as given sets of data. Secondly, we solve the numerical solution $u_{j}^{n}$ in (9) by using the above difference schemes. Finally, we utilize the least squares technique to optimally adapt this model to the data $u\left(x_{j}, t_{n}\right)$ by determining the parameter values for $v$ and $\mu$. Then the deviation of model and data is minimized by the form

$\arg \min _{v, \mu} \sum_{j=0}^{M} \sum_{n=0}^{N}\left|u\left(x_{j}, t_{n}\right)-u_{j}^{n}\right|^{2}$

where $u_{j}^{n}$ is the difference solution at the grind point. Then, by using the difference numerical solution and the least squares method, the estimated values $E v, E \mu$ of the parameters $v, \mu$ in Eq.(9) are shown in Table 1.

Table 1. The results of parameters estimated by the two difference schemes

\begin{tabular}{cccccc}
\hline Method & $t$ & $E v$ & $v$ & $E \mu$ & $\mu$ \\
\hline $\begin{array}{c}\text { Forward-time } \\
\text { centered-space }\end{array}$ & 0.3 & 1.0125 & 1 & 0.0105 & 0.01 \\
$\begin{array}{c}\text { Upwind } \\
\text { difference }\end{array}$ & 0.3 & 1.4282 & 1 & 0.0104 & 0.01 \\
\end{tabular}

From Figure1 and Figure 3, we can see that the error between the difference decomposition and the exact solution is larger with the increase of $x$ and $t$. Furthermore, the results in Table 1 demonstrate that when $v \gg \mu$, i.e. the convection term is domnating, the error of the difference solution compared with the exact solution is large and the good result of the corresponding parameter estimation is not obtained. Consequently, we should find other validity methods for the parameter estimation in Eq.(9). 


\section{Multivariate linear regression technique for the parameter estimation}

We are looking forward to seeing you at the Conference.All the major problems with the difference solutions of the convection-diffusion equation are associated with the parameter $v$ of the convection term in Eq.(1). Concretely, numerical solutions of the convection dominated problems obtained by the above differences methods are polluted by spurious oscillations which spread over the whole spatial domain. In this Section, we consider the case that the convection-diffusion equation (1) has the convenient analytical solution denoted by [3]

$$
u(x, t)=\frac{A_{0} L_{0}}{\sqrt{L_{0}^{2}+\mu t}} \exp \left[-\frac{\left(x-v t-x_{0}\right)^{2}}{4\left(\mu t+L_{0}^{2}\right)}\right]
$$

for the initial condition of a Gaussian distribution of the substance in an infinite medium

$u(x, 0)=A_{0} \exp \left[-\frac{\left(x-x_{0}\right)^{2}}{4 L_{0}^{2}}\right]$

where $A$ is the initial amplitude, $x_{0}$ is the abscissa of the gravity centre of the profile and $L_{0}$ is a measure of the width of the Gaussian profile. Thus in a perfect solution, the original bump will move off at a constant speed and widen and decrease in amplitude.

The task is to estimate the parameters $v, \mu$ of the convection-diffusion equation with the initial condition (13) by using the analytical solution other than the above differences schemes. Now in order to simplify the (12), we set

$$
A=\frac{A_{0} L_{0}}{\sqrt{L_{0}^{2}+\mu t}}, B=\frac{1}{4\left(\mu t+L_{0}^{2}\right)}, C=v t+x_{0}
$$

Then, the analytical solution (12) can be transformed into a simple form

$u(x, t)=A \exp \left[-B(x-C)^{2}\right]$

which is taken by the logarithm on both sides and another form is obtained as

$\ln u(x, t)=\ln A-B x^{2}+2 B C x-B C^{2}$

If we set $t=t_{n}$, i.e. we only analyse the parameter estimation at time point $t_{n}$, and $Y=u\left(x, t_{n}\right)$, $b_{0}=\ln A-B C^{2}, b_{1}=2 B C, b_{2}=-B, X_{1}=x$ and $X_{2}=x^{2}$, then we obtain

$Y=b_{0}+b_{1} X_{1}+b_{2} X_{2}$

In order to identify the validity of this method, the data set $\left\{u\left(x_{j}, t_{n}\right) \mid j=0,1, \cdots, M, n=0,1, \cdots, N\right\}$ from the analytical solution (12) is utilized to estimate regression coefficients $b_{0}, b_{1}$ and $b_{2}$ in (17) containing the parameters $v$ and $\mu$ in the convection-diffusion by the multivariate linear regression.

Now a special problem, i.e. $v=1 \mathrm{~m} / \mathrm{s}, \mu=0.01 \mathrm{~m}^{2} / \mathrm{s}$ for Eq.(1) is taken from the literature [3] to test this parameter estimation for Eq.(9).

$\frac{\partial u}{\partial t}=-\frac{\partial u}{\partial x}+0.01 \frac{\partial^{2} u}{\partial x^{2}}, \quad 0<x<1, \quad 0<t<1$

and its analytical solution is as (10). Then the results of parameters estimated by the multivariate linear regression are described in Table 2.

Table 2. The results of parameters estimated by the multivariate linear regression at time $\mathrm{t}=0.3$

\begin{tabular}{lccccc}
\hline Parameter & $E b_{0}$ & $E b_{1}$ & $E b_{2}$ & $E v$ & $E \mu$ \\
\hline $\begin{array}{l}\text { Estimated } \\
\text { value }\end{array}$ & -4.1993 & -30.1887 & -75.4717 & 1 & 0.01 \\
& & & & & \\
\hline
\end{tabular}

From Table 2, if the convection-diffusion equation has analytical solution, we can obtain the good results of the parameter estimation. Otherwise we should utilize better technique to numerically solve Eq.(1) to obtain optimal parameter estimated values.

\section{Conclusion}

In this paper, the two difference schemes are applied to solving the one-dimensional convectiondiffusion equation and the numerical solutions are designed to adapt the parameters $v$ and $\mu$ of this model. The estimated values of the two parameters show that the multivariate linear regression applied to the analytical solution is better than the difference methods used to estimate the parameters. Of course, better approximation solution to Eq.(1) developed can improve the accuracy of the parameters estimated. Finally, the techniques of the parameter estimation can be utilized to optimally adapt other mathematical models to given data by determining their parameter values such that the deviation of model and data is minimized in a suitable norm.

\section{Acknowledgements}

This work is funded by Fundamental and Advanced Research Project of Chongqing CSTC of China, the project No. is cstc2019jcyj-msxmX0386.

\section{References:}

[1] J. D. Murray, Mathematical Biology: I. An Introduction, Interdisciplinary Applied 
Mathematics. Springer, New York, NY, USA, 3rd edition, 17(2002).

[2] M.Y Kima, M.F. Wheelerb, A multiscale discontinuous Galerkin method for convectiondiffusion-reaction problems. Computers and Mathematics with Applications, 68(2014), 2251-2261.

[3] A. Mohammadi, Numerical solution of the onedimensional advection-diffusion equation using simultaneously temporal and spatial weighted parameters. Australian Journal of Basic and Applied Sciences, 5 (2011), 1536-1543.

[4] G.D. Thiart, Finite difference scheme for the numerical solution of fluid flow and heat transfer problems on nonstaggered grids. Numerical Heat Transfer, 17(1990), 43-62.

[5] J. Bear, Dynamics of Fluid in Porous Media. Elsevier, New York, 1970.

[6] M.T.van Genuchten and W.J.Alves, Analytical solutions of the one-dimensional convectivedispersive solute transport equation. USDA Tech. Bull. 1661,1982.

[7] F.Badrot-Nico, F. Brissaud, V. Guinot, A finite volume upwind scheme for the solution of the linear advection-diffusion equation with sharp gradients in multiple dimensions. Advances in Water Resources, 30 (2007) 20022025.

[8] M. Dehghan, Weighted finite difference techniques for one dimensional advectiondifffusion equation. Appl.Math.Comput., 147(2004), 307-319.

[9] J. Isenberg and C. Gutfinger, Heat transfer to a draining film. International Journal of Heat and Mass Transfer, 16 (1973), 505-511.

[10] H. Karahan, Unconditional stable explicit finite difference technique for the advection-diffusion equation using spreedsheets. Adv. Eng. Sorftware, 38 (2007), 80-84.

[11] D. Liang and W. Zhao, A high-order upwind method for the convection-diffusion problem. Comput. Methods Appl. Mech. Engrg. 147 (1997), 105-115.

[12] M. Dehghan, On the numerical solution of the one dimensional convection-diffusion equation. Mathematical Problems in Engineering, 1(2005), 61-73.

[13] A. Golbabai and M. Javidi, A spectral domain decomposition approach for the generalized Burger's-Fisher equation, Chaos, Solitons \& Fractals, 1(2009), 385-392.

[14] A. Kumar, D. K. Jaiswal and N. Kumar, Analytical Solutions to One-Dimensional Advection-Diffusion Equa-tion with Variable
Coefficients in Semi-Infinite Media, Journal of Hydrology, 380 (2010), 330-337.

[15] A. Kumar, D. K. Jaiswal and N. Kumar, Analytical So-lutions of One-Dimensional Advection-Diffusion Equation with Variable Coefficients in a Finite Domain, Journal of Earth System Science, 118 (2009), 539-549.

[16] Xiaoyang Zheng, Zhengyuan Wei, Discontinuous Legendre Wavelet Galerkin Method for One-Dimensional Advection-Diffusion Equation, Applied Mathematics, 6(2015), 1581-1591.

\section{Creative Commons Attribution License 4.0 (Attribution 4.0 International, CC BY 4.0)}

This article is published under the terms of the Creative Commons Attribution License 4.0

https://creativecommons.org/licenses/by/4.0/deed.en US 